\title{
A QUALIDADE AVALIATIVA DOS CENTROS DE COMPRAS DO POLO DE CONFECÇÕES DO AGRESTE DE PERNAMBUCO
}

\author{
Lourival Lopes Costa Filho (1); \\ Cíntia Amorim (2). \\ (1) Universidade Federal de Pernambuco | CAA | Doutor em Desenvolvimento Urbano \\ e-mail: lourivalcosta@yahoo.com \\ (2) Universidade Federal de Pernambuco | CAC | Especialista \\ e-mail: cintia.r.amorim@gmail.com
}

\begin{abstract}
RESUMO
A pesquisa apresentada visa avaliar a qualidade externa percebida dos centros de compras do Polo de Confecções do Agreste de Pernambuco. A Teoria das Facetas foi adotada para estruturar a investigação empírica, que utilizou o Sistema de Classificações Múltiplas para a coleta de dados e a técnica não paramétrica e multidimensional Análise da Estrutura de Similaridade para analisá-los. 0 resultado da análise dos dados pode servir de referência para a melhoria da qualidade de projetos do ambiente construído e, nesse sentido, alinha-se com os interesses da ergonomia.
\end{abstract}

\section{ABSTRACT}

The research presented aims to evaluate the perceived external quality of shopping malls of the Polo de Confecções do Agreste de Pernambuco (a clothes retail cluster). Facet Theory was adopted to structure the empirical research, using the Multiple Sorting Procedure to collect data and nonparametric procedures and multi-dimensional Similarity Structure Analysis (SSA) to evaluate it. The results of the data analysis can serve as a basis for improving the quality of projects in the built up environment and, accordingly, is aligned with the interests of ergonomics.

\section{INTRODUÇÃO}

A confecção, atualmente, é considerada uma atividade de extrema importância para o agreste pernambucano, gerando e mantendo grande número de empregos formais e informais. Pesquisa realizada pela FADE/UFPE (2003), estimou que, em média, 45 mil pessoas visitaram semanalmente às grandes feiras de confecções populares realizadas nos Centros de Compras de Santa Cruz do Capibaribe, Caruaru e Toritama.

Os centros de compras das três principais cidades do Polo de Confecções do Agreste de Pernambuco atraem consumidores de todo o Brasil, em busca principalmente de artigos de vestuário no atacado e no varejo. Esta pesquisa selecionou para estudo o Polo Caruaru e a Fábrica da Moda, da cidade de Caruaru, o Parque das Feiras, de Toritama, e o Moda Center Santa Cruz, de Santa Cruz do Capibaribe. O Polo Caruaru tem uma estrutura com $64 \mathrm{mil} \mathrm{m}^{2}$ de área coberta, dividida em quatro módulos, dos quais 3 abrigam 500 espaços para lojas e 
o outro reúne grandes empreendimentos. São 3 mil vagas de estacionamento para carros, motos e ônibus. A Fábrica da Moda tem $16 \mathrm{mil} \mathrm{m}^{2}$ de área construída, com 180 lojas e estacionamento com capacidade para 450 automóveis no subsolo. O Parque das Feiras é um complexo com nove hectares que comporta 700 boxes e lojas, além de estacionamento para 2 mil veículos. O Moda Center é o maior centro atacadista de confecções do Brasil, reunindo mais de 10 mil pontos comerciais e estacionamento para 6 mil veículos.

As características visuais do entorno têm importante impacto sobre a experiência humana, podendo evocar fortes emoções como prazer ou medo. Pode nos conduzir a fazer deduções sobre lugares e pessoas a eles relacionadas. Pode influenciar o nosso comportamento, nos levando a evitar ou ir a certos locais. Todos nós respondemos aos estímulos visuais, mas também às propriedades não visuais dos lugares. Por isso, a qualidade visual do ambiente de cada indivíduo tem um poderoso efeito sobre a sua experiência. Quando se considera à aparência do espaço urbano, essa experiência individual deve ser multiplicada pelo grande número de pessoas que o experiencia. Isso significa que, pela condição de imagem pública, sua aparência representa um fenômeno humano-ambiente agregado (NASAR, 2000).

Este artigo aborda a aparência de lugares que podem ser visualizados do espaço público. Mais precisamente, centra-se na avaliação da qualidade externa percebida dos centros de compras do Polo de Confecções do Agreste de Pernambuco. Destaca-se que a aparência, por si, seja insuficiente para tal. É a percepção humana e a avaliação da aparência que lhes dão sentido. A ênfase na aparência não implica, contudo, a desconsideração de outros fatores que contribuem para os locais serem bem-sucedidos. Em vez disso, sugere-se que as decisões sobre a aparência também têm impactos importantes na experiência ergonômica, e que se deve usar o conhecimento científico para informar sobre tais decisões.

Sendo assim, tem-se como objetivo avaliar a qualidade externa percebida dos centros de compras do Polo de Confecções do Agreste de Pernambuco, e medi-la apropriadamente.

Para tal, apresenta uma breve revisão teórica sobre a qualidade visual percebida de lugares, destaca algumas considerações teórico-metodológicas que justificam a adoção da Teoria das Facetas como base estrutural da pesquisa empírica, introduz questões metodológicas que selecionou o Sistema das Classificações Múltiplas como instrumento para a coleta dos dados e a técnica não paramétrica e multidimensional Análise da Estrutura de Similaridade para interpretá-los. Conclui-se, apresentando os resultados das análises dos dados.

\section{CONTEÚDO}

O ambiente construído pode evocar sentimentos extremos e intensos, mas também geralmente provoca alterações afetivas menores. Este artigo considera toda gama de respostas afetivas ou emocionais para esse tipo de espaço artificial, incluindo aquelas mais extremas, às vezes chamadas de respostas estéticas. Conforme Nasar (2000), a avaliação afetiva implica numa atribuição ao ambiente construído, tal como julgamentos dos indivíduos que eles gostam de um determinado lugar ou que certo tipo de pessoa vive lá.

Estudar a qualidade avaliativa de lugares justifica-se para a área da ergonomia, uma vez que seus efeitos no comportamento espacial têm importância para aplicação na ergonomia do ambiente construído, devido ao interesse dessa disciplina em compreender padrões da atividade humana no espaço. Para o estabelecimento de diretrizes que tornem os ambientes agradáveis aos seus usuários, a qualidade avaliativa de lugares tem também aplicação direta para os profissionais que projetam os ambientes.

Esses profissionais, infelizmente, muitas vezes ignoram ou subestimam valores populares, produzindo, assim, lugares pouco convidativos. As diferenças vão além das diferenças de intensidade de preferência. O que arquitetos gostam, o público desgosta, e o que o público gosta, arquitetos desgostam (NASAR, 2000; NASAR, 1988; GROAT, 1982). 
A qualidade visual percebida é um constructo psicológico, na medida em que envolve avaliações subjetivas. Cada avaliação tem uma referência primária tanto para o ambiente como para os sentimentos das pessoas sobre tal. Os primeiros são chamados de julgamentos perceptuais/cognitivos, e os segundos de julgamentos emocionais. Embora a qualidade visual percebida dependa, em parte, de fatores perceptuais/cognitivos, é, por definição, um julgamento emocional que envolve avaliação e sentimentos (NASAR, 1988). Como resultado, a qualidade visual percebida será medida aqui através dos julgamentos avaliativos dos centros de compras do Polo de Confecções do Agreste de Pernambuco.

Para serem relevantes, esses julgamentos devem centrar-se nas dimensões emocionais que as pessoas realmente usam para avaliar os lugares. Os psicólogos Ward e Russel (1981) examinaram tais questões e, usando uma variedade de estratégias de pesquisa e medidas, identificaram quatro aspectos de avaliação ambiental - agradabilidade, excitante, emocionante, relaxante - como sendo os mais salientes. A agradabilidade é uma dimensão puramente avaliativa, já a excitação independe de avaliação. De acordo com a visão de Nasar (1988 e 2000) diferentes combinações de agradabilidade e excitação produzem emoção (interesse-desinteresse) e relaxamento (tranquilo-intranquililo). Dentro dessa perspectiva, por exemplo, um lugar emocionante seria mais excitante e agradável do que um desinteressante; enquanto um lugar relaxante seria mais agradável e menos excitante do que um intranquilo.

Muito embora as respostas avaliativas, por si só, não podem prever o comportamento real, a avaliação combinada de respostas avaliativas e o comportamento esperado dão uma boa indicação do comportamento real. Sob esse prisma, nesta pesquisa, os entrevistados foram solicitados a indicar o grau em que eles gostariam de visitar, comprar ou passar algum tempo nos principais centros de compra do Polo de Confecções do Agreste de Pernambuco.

\section{QUESTÕES TEÓRICO-METODOLÓGICAS}

Esta pesquisa adota a Teoria das Facetas, desenvolvida por Louis Guttman nos anos 50 do século passado, no desenho da investigação empírica, assim como para evidenciar a descrição de um sistema de categorias e seus elementos internos capazes de descrever o problema a ser estudado, as hipóteses formuladas e, mais especificamente, para ajudar na construção dos instrumentos de coleta e de análise dos dados (BILSKY, 2003; SHYE; ELIZUR; HOFFMAN, 1994).

A Teoria das Facetas postula que uma boa teoria, ou um bom modelo teórico, deve se refletir na estrutura dos dados empíricos, ou seja, que a estrutura teórica construída pelo pesquisador seja encontrada na estrutura dos dados empíricos graficamente apresentados (COSTA FILHO, 2014).

A partir do exposto, o uso da Teoria das Facetas envolve inicialmente a identificação dos diferentes conceitos ou dimensões que delineiam a pesquisa. Tal conhecimento prévio pode advir da literatura sobre o assunto, onde relações são apontadas, ou de explorações in loco.

As relações entre os diversos aspectos da experiência das pessoas com um determinado ambiente podem ser sumarizadas através da sentença estruturadora (mapping sentence), instrumento básico da Teoria das Facetas, que descreve com precisão os componentes físicos dos ambientes e a forma como eles são vivenciados pelos usuários.

A Tabela 1 apresenta a sentença estruturadora geral para a avaliação dos centros de compras do Polo de Confecções do Agreste de Pernambuco, em que se diferenciam três tipos de facetas: o primeiro tipo (background) se refere aos grupos pesquisados. O segundo tipo diz respeito ao conteúdo das variáveis pesquisadas (conteúdo). Juntas, as facetas de background e a de conteúdo determinam o campo de interesse da pesquisa (domínio). 0 terceiro tipo de faceta equivale ao universo de respostas, apresentada como numa escala ordenada de aceitação (racional). 
Tabela 1 | Sentença Estruturadora para a avaliação da qualidade externa percebida nos centros de compras do Polo de Confecções do Agreste de Pernambuco

\begin{tabular}{|c|c|c|c|}
\hline \multicolumn{4}{|c|}{$\begin{array}{l}\text { Em que medida a pessoa x (consumidor - projetistas) avalia que a aparência externa de um centro de } \\
\text { compras do Polo de Confeç̧ões do Agreste com }\end{array}$} \\
\hline $\begin{array}{l}\text { (Faceta A) } \\
\text { VITRINE EXTERNA, } \\
\text { (A1) presente } \\
\text { (A2) ausente }\end{array}$ & $\begin{array}{l}\text { (Faceta B) } \\
\text { MíDIA EXTERIOR, } \\
\text { (B1) de identificação do centro } \\
\text { (B2) mista (centro/lojas) }\end{array}$ & $\begin{array}{l}\text { (Faceta C) } \\
\text { ESTACIONAMENTO } \\
\text { (C1) no nível da rua } \\
\text { (C2) no subsolo }\end{array}$ & favorece \\
\hline $\begin{array}{l}\text { (Racional) } \\
\text { (1) nada; } \\
\text { (2) pouco; } \\
\text { (3) mais ou menos; } \\
\text { (4) muito; } \\
\text { (5) muitíssimo }\end{array}$ & \multicolumn{3}{|c|}{$\begin{array}{l}\text { o seu desejo de visitar, comprar e passar tempo nesse lugar? } \\
\text { (uma expressão da qualidade externa percebida) }\end{array}$} \\
\hline
\end{tabular}

Fonte | autores do artigo com base nos resultados da pesquisa

Para expressar o assunto de pesquisa nos termos da Teoria das Facetas, é necessário definir várias facetas e os seus elementos internos. Nesse sentido, a população amostral abordada foi constituída por dois diferentes tipos de visões sobre o ambiente construído. Uma delas é especializada no assunto, fundamentada na experiência científica, enquanto outra é não especializada, marcada pelo senso comum: 1| consumidor; 2| projetistas.

O objetivo desta pesquisa é avaliar a qualidade externa percebida dos centros de compras do Polo de Confecções do Agreste de Pernambuco. Daí, três facetas de conteúdo VITRINE EXTERNA, MÍDIA EXTERIOR, ESTACIONAMENTO - foram consideradas como importantes referentes do ambiente construído para tal tipo de avaliação. A partir da diversidade em que aparecem na área externa desses centros, a Faceta A, VITRINE EXTERNA, pode estar: (A1) presente ou (A2) ausente. A Faceta B, MÍDIA EXTERIOR, define a possibilidade de o anúncio ser apenas (B1) de identificação do centro ou (B2) mista (tanto do centro como das lojas). A Faceta C, ESTACIONAMENTO, diz respeito a sua localização (C1) no nível da rua e (C2) no subsolo.

As combinações dos elementos internos das facetas de conteúdo (A2 x B2 x C2) totalizam oito (8) conjuntos de diferentes situações a serem avaliadas. Nesta pesquisa, entretanto, foi considerado apenas as situações relacionadas aos objetos de estudos empíricos, reduzindo para quatro (4) o número de combinações possíveis de serem encontradas nesses locais.

A Faceta de RACIONAL propõe cinco intervalos de escolha para as respostas dos sujeitos: 1| nada; 2| pouco; 3| mais ou menos; 4| muito; 5| muitíssimo.

A sentença estruturadora para a avaliação da qualidade externa dos centros de compras do Polo de Confecções do Agreste de Pernambuco, como uma referência inicial da pesquisa, é analisada em relação aos resultados empíricos que devem confirmar ou refutar as hipóteses levantadas. Logo, após a análise dos dados, há informações suficientes para construir ou não uma nova sentença estruturadora como consequência direta dos resultados.

\section{QUESTÕES METODOLÓGICAS}

Como instrumento para a coleta de dados, a pesquisa fez uso do Sistema de Classificações Múltiplas (SCM). O procedimento, segundo Costa Filho (2014), consiste em solicitar ao participante que agrupe os elementos, de acordo com suas similaridades, em função de critérios livres (classificações livres) ou estabelecidos (classificações dirigidas). O SCM permite o uso de imagens, elementos difíceis de serem acomodadas em outros métodos. 
Nesta pesquisa foram utilizadas apenas classificações dirigidas, em que os entrevistados foram solicitados a classificar os elementos apresentados de acordo com o grau que eles favorecem o seu desejo de visitar, comprar e passar tempo, com o objetivo de avaliar a qualidade externa percebida dos centros de compras do Polo de Confecções do Agreste de Pernambuco, além de medi-los adequadamente.

Como elementos de estímulo, optou-se por utilizar fotografias da aparência externa dos centros de compras enfocados. Assim sendo, conforme explicado anteriormente, obteve-se quatro fotografias que expressam o modo como os elementos que definem a investigação se relacionam (Figura $1 \mathrm{a} / \mathrm{d}$ ). Para as classificações, foram apresentadas aos participantes fotografias coloridas e impressas no tamanho $13 \times 18 \mathrm{~cm}$.

Figura 1 | Elementos de Estímulo Apresentados para as Classificações

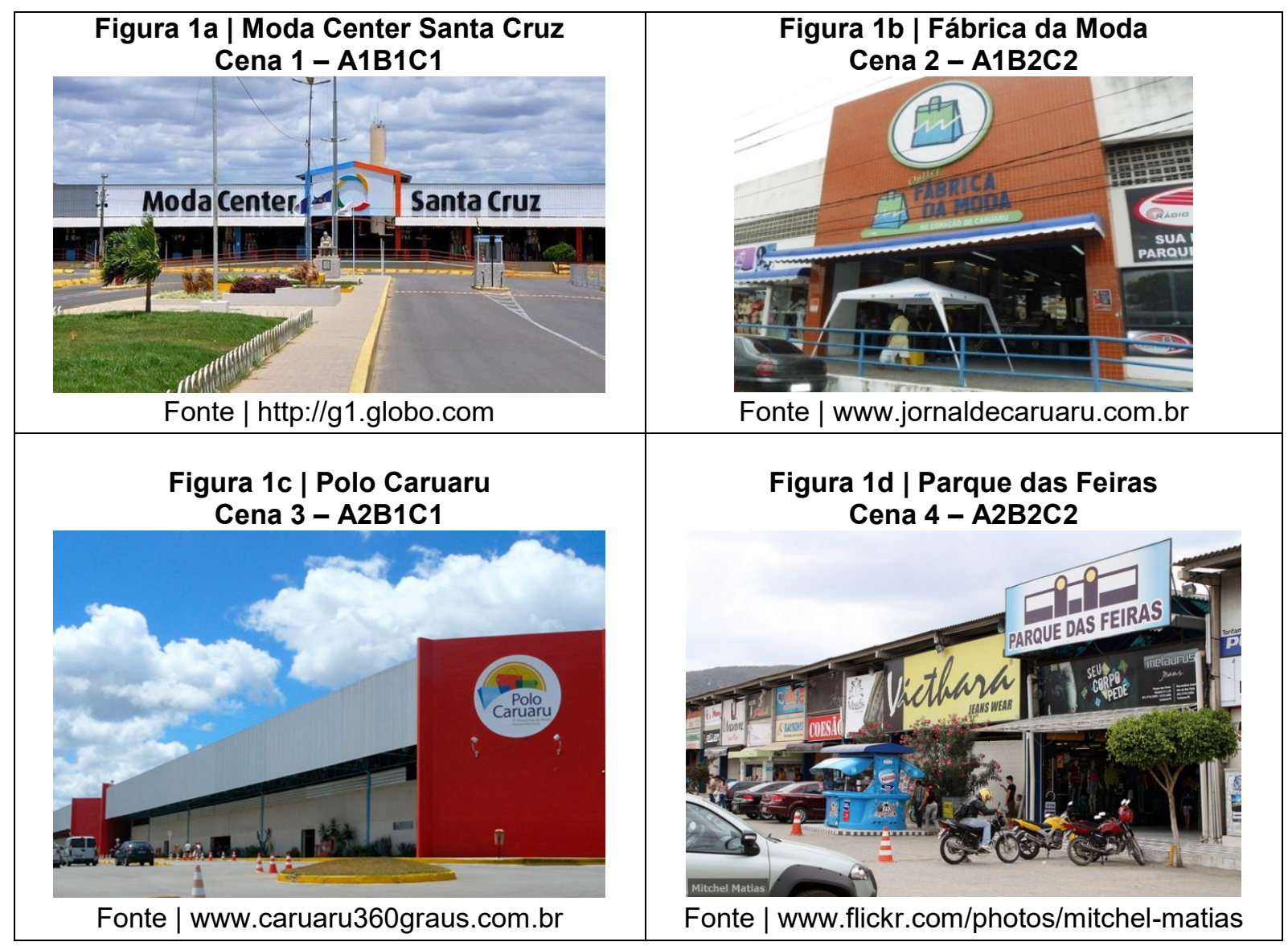

Os dados obtidos nas classificações dirigidas foram analisados através da técnica estatística não paramétrica e multidimensional Análise da Estrutura de Similaridade (Similarity Structure Analysis - SSA), realizada com o auxílio do programa informático HUDAP (Hebrew University Data Analysis Package), desenvolvido por Amar e Toledano em 1994.

A SSA é um sistema de escalonamento multidimensional concebido para analisar a matriz de correlações entre " $n$ " variáveis representadas graficamente como pontos num espaço euclidiano. O sistema fundamenta-se no princípio da contiguidade que, como tal, traduz as relações de similaridades entre itens, configurado pelas distâncias entre os pontos. Isso significa que a proximidade das variáveis no espaço multidimensional é proporcional ao grau de correlação que apresentam. Essas relações de similaridades podem formar regiões de contiguidade que possibilitam verificar se as hipóteses iniciais são transformadas em 
hipóteses regionais, em relação às quais se espera evidenciar regiões que abarquem aos elementos internos de cada faceta (ROAZZI; MONTEIRO; RULLO, 2009).

A confirmação das hipóteses regionais revela aspectos relativamente estáveis do conceito investigado, dando-lhe legitimidade, além de demonstrar a estrutura interna de conceitos e atributos, possibilitando a percepção de componentes empiricamente verificáveis e da forma como se inter-relacionam (SHYE; ELIZUR; HOFFMAN, 1994).

Quanto à população amostral, no total, 38 participantes foram submetidos às classificações. Essa amostra englobou 20 consumidores e 18 projetistas de ambientes. No grupo de consumidores, a maioria é do sexo feminino (12), tem entre 50 e 59 anos de idade (9), ensino médio completo (14) e renda de até dois salários mínimos mensais (15). Já no grupo dos projetistas, a maioria é do sexo feminino (12), tem entre 25 e 29 anos de idade (12), ensino superior completo e renda de 4 a 6 salários mínimos mensais (12).

\section{QUESTÕES DE RESULTADOS}

A questão explorada nas classificações dirigidas, que fez uso da SSA para interpretar os dados, diz respeito ao desejo de consumidores/projetistas visitarem, comprarem e passarem tempo nos centros de compras do Polo de Confecções do Agreste de Pernambuco, identificados através de quatro (4) cenas representadas em fotografias, com o objetivo de avaliar a qualidade externa percebida desses centros, além de medi-los apropriadamente.

Os resultados dos dados apresentados nos diagramas da SSA revelam que as três facetas definidas - VITRINE EXTERNA, MÍDIA EXTERIOR, ESTACIONAMENTO - formam estruturas de contiguidade entre os itens similares de mesmo elemento interno, confirmando a aderência desses referentes físicos ambientais para a avaliação da qualidade externa percebida nos centros de compras enfocados, conforme previsto nas hipóteses iniciais.

Através dos diagramas da SSA para as facetas - VITRINE EXTERNA, MÍDIA EXTERIOR, ESTACIONAMENTO - nota-se que os participantes captaram essas três categorias que formam hipóteses regionais. As Figuras 2, 3 e 4 descrevem uma linha que divide os espaços dos diagramas em duas regiões distintas. Essas facetas desempenham papel axial, em que as linhas dão origens a faixas ordenadas, indicando que seus elementos variam segundo uma escala gradativa na preferência dos participantes. Além disso, esse padrão de divisão denota a forte influência dessas categorias para a avaliação da qualidade externa percebida nos centros de compras do Polo de Confecções do Agreste de Pernambuco, conforme previsto nas hipóteses iniciais desta pesquisa.

Na Figura 2, os itens/centros de compras com vitrine externa presente localizaram-se na região inferior do diagrama da SSA, já aqueles com vitrine externa ausente concentraram-se na região superior. Isso significa que foram captados conforme sugerido na hipótese inicial, sendo reconhecidos como tais pelos participantes desta pesquisa. Cabe destacar que, conforme os escores atribuídos a essa faceta/categoria, a faixa com mais qualidade externa percebida é a inferior, com vitrines externas presentes ou centros de compras abertos, e o item/centro de número 01 (Figura 1a) é aquele que melhor traduz essa preferência.

Conforme mostra a Figura 3, os itens/centros de compra com mídia exterior apenas de identificação do centro reuniram-se numa faixa à esquerda do mapa da SSA, já os que exibem tanto indicação do centro como de algumas lojas posicionaram-se à direita. Isso confirma, de modo semelhante à faceta anterior, que tanto essa categoria como os seus elementos internos foram captados pelos respondentes, de acordo com a hipótese inicialmente prevista. A partir dos escores atribuídos pelos participantes desta pesquisa a essa faceta/categoria, a faixa com mais qualidade externa percebida é a da esquerda, com mídia exterior apenas de identificação do centro, e o item/centro de compras que melhor expressa tal opção, assim como na categoria anterior, é o de número 01 (Figura 1a). 
Figura 2 | Teste da Faceta A, Vitrine Externa, sobre o diagrama da SSA Dimensionalidade 3. Eixo 1 versus Eixo 2. C.o. $A=0.00$

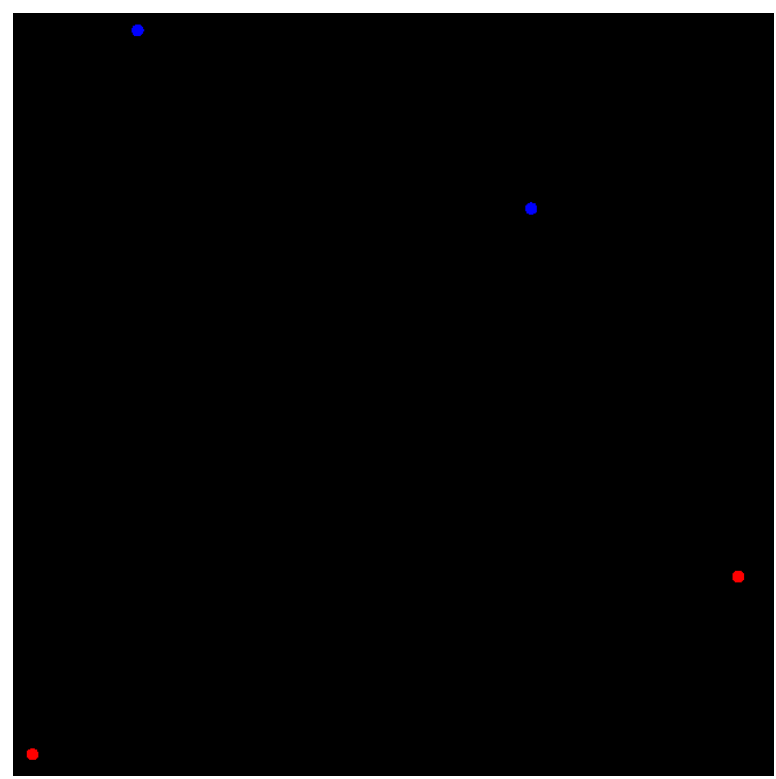

Fonte | autores do artigo com base nos resultados da pesquisa

Figura 3 | Teste da Faceta B, Mídia Exterior, sobre o diagrama da SSA Dimensionalidade 3. Eixo 1 versus Eixo 2. C.o.A = 0.00

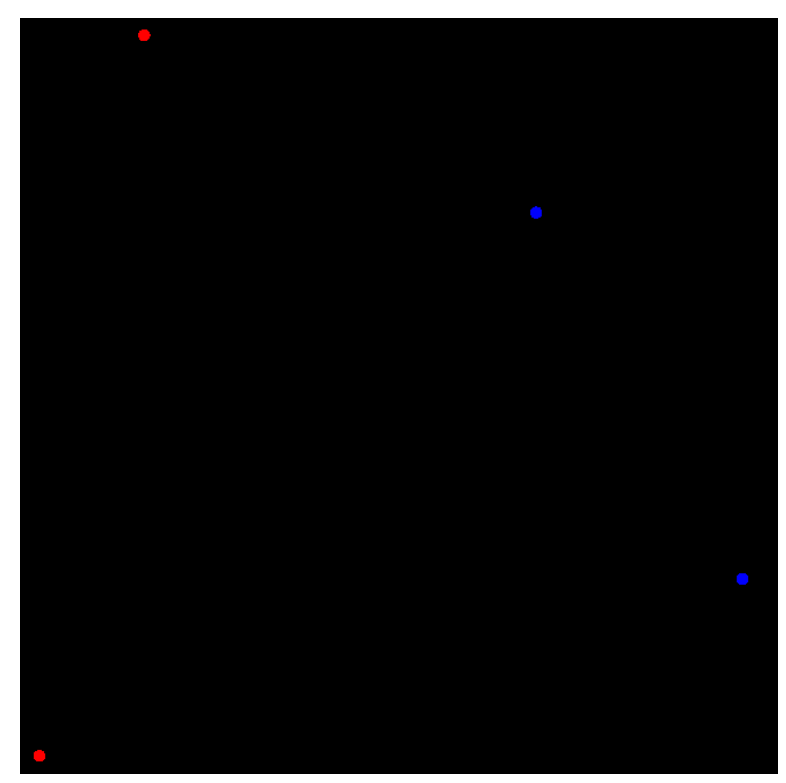

Fonte | autores do artigo com base nos resultados da pesquisa

Os itens/centros de compras com estacionamento no nível da rua estão agrupados na faixa da esquerda do diagrama plotado pela SSA. A faixa da direita mostra o único item/centro, cujo estacionamento não é visível da rua, estando localizado no subsolo (Figura 4). Tais partições, de modo semelhante às anteriores, confirmam que essa categoria é aderente à avaliação dos centros de compras enfocados, conforme inicialmente previsto. Levando-se em conta os escores outorgados pelos participantes às cenas apresentadas, a faixa com mais qualidade externa percebida é a da esquerda, com estacionamentos no nível da rua, e o item/centro de número 01 (Figura 1a) é aquele que melhor exprime esse favorecimento. 
Figura 4 | Teste da Faceta C, Estacionamento, sobre o diagrama da SSA Dimensionalidade 3. Eixo 1 versus Eixo 2. C.o. $A=0.00$

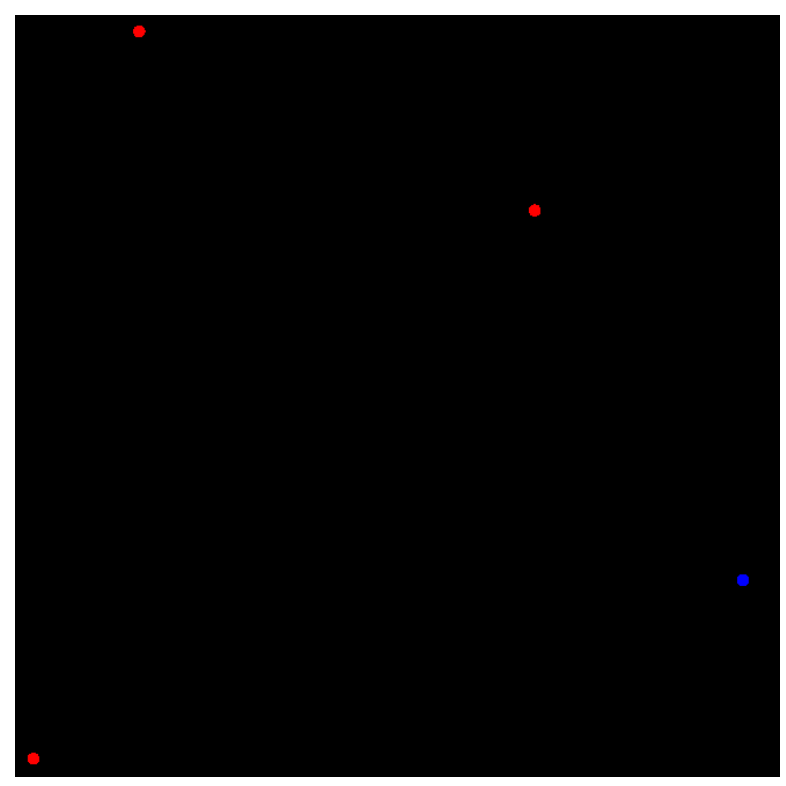

Fonte | autores do artigo com base nos resultados da pesquisa

Conforme evidenciado nas Figuras 2 e 3, consumidores e projetistas estão localizados justapostos, sobre as linhas limítrofes que definem as duas regiões nos diagramas da SAA para as categorias de vitrine externa e mídia exterior. Isso significa que os dois grupos, quando avaliados isoladamente, são influenciados tanto pela presença como pela ausência de vitrine externa nos centros, assim como tanto pela mídia exterior ser apenas de identificação dos centros como mista. Há, portanto, consenso entre os grupos para as categorias de vitrine externa e de mídia exterior na avaliação da qualidade externa dos centros de compras enfocados. Já na Figura 4, nota-se que os grupos estão localizados na faixa da esquerda do espaço. Ambos, portanto, são mais influenciados pelo estacionamento no nível da rua. Há, de modo semelhante, consenso entre os grupos para a categoria estacionamento. Cabe, finalmente, destacar que o item que melhor prediz a qualidade externa percebida para as três categorias, na percepção de consumidores e projetistas, é aquele de número 1 (Figura 5).

Figura 5 | Centro com a Melhor Qualidade Externa Percebida

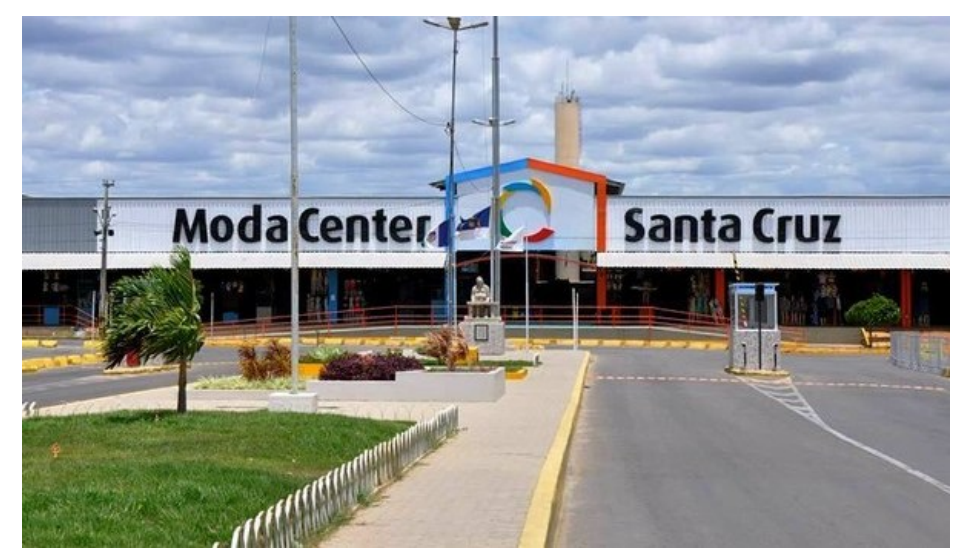

Cena 1 - A1B1C1

Fonte | http://g1.globo.com 
Buscando avaliar a qualidade exterior percebida dos centros de compras do Polo de Confecções do Agreste de Pernambuco, apurou-se que as três categorias/facetas - vitrine externa, mídia exterior, estacionamento - demostraram ser consistentes ou determinantes para o tipo de avaliação proposto, conforme previsto nas hipóteses iniciais desta pesquisa.

Cabe destacar, mais especificamente, que a vitrine externa presente ou o centro aberto, a mídia exterior apenas com a identificação do centro e o estacionamento no nível da rua definem a qualidade externa percebida para os centros de compras enfocados, na visão dos participantes dos dois diferentes grupos abordados.

Ao analisar o consenso dos resultados entre os dois grupos abordados - consumidores e projetistas - apurou-se haver tal condição para todas as categorias/facetas - vitrine externa, mídia exterior, estacionamento - também inicialmente consideradas.

Como consequência direta da análise dos dados, que confirmaram todas as hipóteses inicialmente formuladas, a sentença estruturadora para a avaliação da qualidade externa percebida dos centros de compras do Polo de Confecções do Agreste de Pernambuco foi confirmada/validada em relação às categorias/facetas e a todos os seus elementos internos.

Esta pesquisa revelou resultados que regem respostas avaliativas para as áreas externas dos centros de compras enfocados. O resultado da análise dos dados podem fornecer ainda referências para a melhoria de projetos do ambiente construído, face aos desafios de um novo olhar que sempre deve levar em conta a cognição dos seus usuários.

\section{REFERÊNCIAS}

FADE/UFPE. Estudo de Caracterização Econômica do Polo de Confecções do Agreste Pernambucano. FADE/ UFPE/ SEBRAE: Recife - PE, 2003. Relatório de Pesquisa. Disponível em: $<$ www.sebraepe.com.br>. Acesso em: 23 de junho de 2016.

BILSKY, Wolfgang. A Teoria das Facetas: noções básicas. In Estudos de Psicologia, v.8, n.3, 2003. p. 357-365

COSTA FILHO, Lourival. O Enfoque da Teoria das Facetas na Avaliação de Lugares. In: MONT'ALVÃO, C.; VILLAROUCO, Vilma (Orgs.), Um Novo Olhar para o Projeto 2: a ergonomia no ambiente construído. Recife: Editora UFPE, 2014. p. 11 - 26.

GROAT, Linda. Meaning in Post-Modern architecture. An examination using the multiple sorting task. In Journal of Environmental Psychology, v. 2, n. 1, March 1982. p. 3 - 22.

NASAR, Jack. Environments Aesthetics: theory, research, and applications. New York: Cambridge University Press, 1988.

NASAR, Jack. The Evaluative Image of the City. London: SAGE, 1998.

ROAZZI, Antonio; MONTEIRO, Circe Maria Gama; RULLO, Giuseppina. Residencial satisfaction and place attachment: A cross-cultural investigation. In Arie Cohen (Ed.). Facet Theory and Scaling: In search of structure in behavioral and social sciences. Israel: Rubin R. I. D, 2009.

SHYE, Samuel; ELIZUR, Dov; HOFFMAN, Michel. Introduction to Facet Theory: Content design and intrinsic data analysis in behavioral research. London: Sage, 1994.

WARD, Larry; RUSSEL, James A. The psychological representation of molar environments. In Jornal of Experimental Psychology: General, 110, 1981. p. 121 - 152. 